\title{
Surgical Outcomes and Safety of Robotic Sacrocolpopexy in Women With Apical Pelvic Organ Prolapse
}

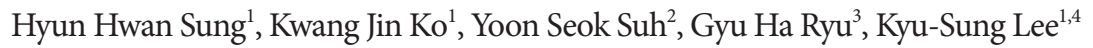 \\ ${ }^{1}$ Department of Urology, Samsung Medical Center, Sungkyunkwan University School of Medicine, Seoul, Korea \\ ${ }^{2}$ Department of Urology, Center for Prostate Cancer, Research Institute and Hospital of National Cancer Center, Goyang, Korea \\ ${ }^{3}$ Office of R\&D Strategy \& Planning, Samsung Medical Center, Seoul, Korea \\ ${ }^{4}$ Department of Medical Device Management \& Research, SAIHST, Sungkyunkwan University, Seoul, Korea
}

Purpose: This study aimed to investigate the surgical outcomes and safety of robotic sacrocolpopexy (RSC) in patients with uterine/vaginal vault prolapse.

Methods: Between January 2009 and June 2015, 16 women with apical prolapse underwent RSC. Pelvic organ prolapse quantification (POP-Q) examination was performed, and treatment success was defined as the presence of grade 0 or I apical prolapse upon POP-Q examination at the final follow-up. Pelvic floor distress inventory-short form 20 (PFDI-SF 20) was administered at every follow-up. A treatment satisfaction questionnaire was administered by telephone to evaluate patient satisfaction with the operation.

Results: Median age was 65 years (interquartile range [IQR], 56-68 years), and follow-up duration was 25.3 months (IQR, 5.4-34.0 months). Thirteen women (81.3\%) had $\geq$ grade III apical prolapse. Operation time was 251 minutes (IQR, 236-288 minutes), and blood loss was $75 \mathrm{~mL}$ (IQR, 50-150 mL). Median hospital stay was 4 days (IQR, 3-5 days). At the final followup, treatment success was reported in all patients, who presented grade $0(n=8,57.1 \%)$ and grade $I(n=6,42.9 \%)$ apical prolapse. Dramatic improvements in PFDI-SF 20 scores were noted after RSC (from 39 to 4; $\mathrm{P}=0.001$ ). Most patients (12 of 13) were satisfied with RSC. An intraoperative complication (sacral venous plexus injury) was reported in 1 patient, and there was no conversion to open surgery. Mesh erosion was not reported.

Conclusions: RSC is an efficient and safe surgical option for apical prolapse repair. Most patients were satisfied with RSC. Thus, RSC might be one of the best treatment options for apical prolapse in women.

Keywords: Pelvic Organ Prolapse; Uterine Prolapse; Robotic Sacrocolpopexy; Robotic Surgical Procedures

- Grant/Fund Support: This research was supported by a grant of the Korea Health Technology R\&D Project through the Korea Health Industry Development Institute (KHIDI), funded by the Ministry of Health \& Welfare, Republic of Korea (grant number: HI14C3229).

- Research Ethics: This study was performed with the approval of the Institutional Review Board of Samsung Medical Center at Sungkyunkwan

University School of Medicine (approval number: 2016-03-163).

- Conflict of Interest: No potential conflict of interest relevant to this article was reported.

\section{INTRODUCTION}

Pelvic organ prolapse (POP) is a common, troublesome, and costly problem in women. In the United States (US), the cumulative incidence of POP surgery was reported to be $12.6 \%$, and the age-specific annual risk of POP was found to increase pro-

Corresponding author: Kyu-Sung Lee (iD http://orcid.org/0000-0003-0891-2488 Department of Urology, Samsung Medical Center, Sungkyunkwan University School of Medicine, 81 Irwon-ro, Gangnam-gu, Seoul 06351, Korea E-mail: ksleedr@gmail.com / Tel: +82-2-3410-3559 / Fax: +82-2-3410-3027 Submitted: May 23, 2016 / Accepted after revision: August 15, 2016 ative Commons Attribution Non-Commercial License (http://creativecommons.org/licenses/by-nc/4.0/) which permits unrestricted non-commercial use, distribution, and reproduction in any medium, provided the original work is properly cited. 
gressively until the seventh decade of life when the annual risk is 4.3 per 1,000 women [1]. Routine pelvic examinations have shown that the prevalence of any degree of POP increases up to one-third in women aged $20-59$ years, because mild prolapse is common and frequently asymptomatic [2,3]. In addition, one study reported that the estimated number of women with POP will increase from 3.3 million in 2010 to 4.9 million in 2050 in the US [4]. The total annual cost of POP surgery in the US is estimated to be over US $\$ 1$ billion [5]. Indirect costs related to time off from work and loss of wages and the negative impact on the quality of life in patients should also be considered [6].

Abdominal sacrocolpopexy (ASC) was introduced in 1962 [7] and has been the gold standard of treatment for uterine/ vaginal vault prolapse (apical prolapse) for over 50 years $[8,9]$. However, this approach has some shortcomings related to the use of laparotomy, such as a high morbidity rate and long hospital stay. On the other hand, laparoscopic sacrocolpopexy (LSC) has been reported to have some advantages over ASC, including minimal invasiveness, reduced blood loss, reduced postoperative pain, shorter duration of recovery, and earlier return to normal activity [10-12]. However, laparoscopic surgery is technically challenging and can present a steep learning curve for surgeons. In addition, postural ergonomic strain during LSC is bothersome to surgeons [13].

Robotic sacrocolpopexy (RSC) was first described by Di Marco et al. [14] in 2004. Since its introduction, RSC has proven to be a reliable, efficient, and safe surgical option for uterine/ vaginal vault prolapse repair [15-17]. RSC has the advantages of being minimally invasive and having a shorter learning curve than LSC. However, data on the surgical technique and measures of success are not consistent [16]. Although a few studies on sacrocolpopexy have been performed in Korea, data on RSC in Korean patients have not been reported to date $[18,19]$. Therefore, the aim of this study was to introduce RSC at one institution and to examine the surgical outcomes and safety of RSC in patients with apical prolapse.

\section{MATERIALS AND METHODS}

\section{Patients}

Between January 2009 and June 2015, 16 women with symptomatic stage II or greater uterine/vaginal vault prolapse underwent RSC at a tertiary hospital. Data on patient age, body mass index (BMI), operation history, American Society of Anesthesiologists (ASA) physical status classification, estimated blood loss, operation time, length of hospital stay, surgical outcomes, and perioperative complications were retrospectively obtained from patient medical records and telephone surveys. In all patients receiving uterus-preserving RSC, gynecologic evaluation was done preoperatively.

\section{Surgical Technique}

The surgical technique performed at the institution has been described briefly in a previous study [20]. Patients were placed in a dorsal lithotomy and steep Trendelenburg position $\left(30^{\circ}\right)$. A Foley catheter was placed, pneumoperitoneum was created, and 5 laparoscopic ports were placed to form a " $W$ " shape (one $12-\mathrm{mm}$ trocar for the camera port above the umbilicus, three $8-\mathrm{mm}$ trocars for the robotic arms, and one $12-\mathrm{mm}$ trocar for the assistant). A $0^{\circ}$ or $30^{\circ}$ lens was used interchangeably. A vaginal retractor was used to push up the vaginal wall anteriorly, and then, the peritoneum of the uterovesical junction was dissected. A peritoneal dissection between the posterior uterus and vagina was performed to carry out peritoneal tunneling at the lateral level of the broad ligament opening. A peritoneal incision was made over the sacral promontory at the bifurcation of the aorta, and sacral dissection was performed until the anterior longitudinal ligament was exposed. An anterior mesh $(4 \times$ 5-cm nonabsorbable polypropylene monofilament mesh; Gynemesh, Gynecare, Ethicon Inc., Somerville, NJ, USA) was placed and sutured with nonabsorbable sutures (Ethibond, Ethicon Inc.). The anterior mesh was not used in the case of a small-sized uterus. A T-shaped mesh was placed on the posterior dissection plane, and both arms of the mesh were drawn through the peritoneal tunnel of the broad ligament. The anterior and posterior meshes were sutured together on the anterior side of the uterus. In the case of vaginal vault prolapse, peritoneal dissection of the proximal vagina was performed, and then a Y-shaped mesh was placed. The tail of the T- or Y-shaped mesh was fixed with the anterior longitudinal ligament on the sacral promontory. Next, vaginal examination was performed to assess the apical support of the vagina. The peritoneum was then reapproximated over the mesh with absorbable sutures [20]. Transobturator midurethral sling surgery was performed after RSC in patients with stress incontinence triggered by heaving, climbing, or patient preference $(n=8,50.0 \%)$. Staging of stress incontinence was evaluated after reduction of apical prolapse. In case of the presence of central-type cystocele, transvaginal cystocele repair was performed using a transobturator four-arm mesh $(n=1,6.3 \%)$. Vaginal packing was placed and 
then removed on postoperative day 1.

\section{Follow-Up}

Patients were routinely discharged on postoperative days 2-5. The patients visited the outpatient clinic at $1,3,6$, and 12 months after the procedure and then at least once a year. The POP quantification (POP-Q) examination was performed preand postoperatively and at every follow-up. The pelvic floor distress inventory-short form 20 (PFDI-SF 20) was administered preoperatively and at every follow-up. The PFDI-SF 20 is a disease-specific questionnaire that evaluates the symptoms of pelvic floor disorders and has been validated and translated into Korean [21]. This questionnaire included 4 subscales: total score inventory, POP distress inventory, colorectal-anal distress inventory, and urinary distress inventory. A five-tier treatment satisfaction questionnaire (TSQ) was administered by telephone to evaluate patient satisfaction with the operation.

\section{Data Analysis}

The primary endpoint of the present study was to evaluate the surgical outcome of RSC. Treatment success was defined as the presence of grade 0 or I apical prolapse on the POP-Q examination at the final follow-up. To measure the subjective outcomes, the preoperative and postoperative PFDI-SF 20 scores were

Table 1. Baseline characteristics $(n=16)$

\begin{tabular}{lc}
\hline Variable & Value \\
\hline Age $(\mathrm{yr})$ & $65(56-68)$ \\
Body mass index $\left(\mathrm{kg} / \mathrm{m}^{2}\right)$ & $25.4(24.0-28.3)$ \\
Follow-up duration $(\mathrm{mo})$ & $25.3(5.4-34.0)$ \\
ASA PS classification, grade & II (I-III) \\
Previous abdominal surgery & $6(37.5)$ \\
Hysterectomy & $5(31.3)$ \\
Salpingo-oophorectomy & $1(6.3)$ \\
Previous POP repair & $2(12.5)$ \\
Stress incontinence & $11(68.8)$ \\
Grade I & $7(43.8)$ \\
Grade II & $4(25.0)$ \\
Previous anti-incontinence surgery & $2(12.5)$ \\
Uterine/vaginal vault prolapse & \\
Grade II & $3(18.8)$ \\
Grade III & $10(62.5)$ \\
Grade IV & $3(18.8)$
\end{tabular}

Values are presented as median (interquartile range) or number (\%). ASA PS, American Society of Anesthesiologists physical status; POP, pelvic organ prolapse. compared. The secondary endpoint was to assess the surgical safety of this technique and evaluate patient satisfaction with the operation. Operation time from skin incision to closure, estimated blood loss, intraoperative complications, incidence of open conversion, and length of hospital stay were evaluated. Postoperative complications were graded using the ClavienDindo classification system.

\section{Statistical Analysis}

Data are presented as counts and percentages. Continuous variables are expressed as median and interquartile range (IQR). Data were statistically analyzed using the Wilcoxon signedrank nonparametric test for matched pairs. A 2-sided P-value of $<0.05$ was considered statistically significant. All statistical analyses were performed using IBM SPSS Statistics Version 22.0 (IBM Co., Armonk, NY, USA).

\section{RESULTS}

The median age was 65 years (IQR, 56-68 years), and median follow-up duration was 25.3 months (5.4-34.0 years). The median ASA physical status classification grade was 2 (1-3), and BMI was $25.4 \mathrm{~kg} / \mathrm{m}^{2}\left(24.0-28.3 \mathrm{~kg} / \mathrm{m}^{2}\right)$. A history of abdominal surgery was reported in 6 patients (37.5\%), including hysterectomy $(\mathrm{n}=5,31.3 \%)$. Two patients (12.5\%) had previously undergone cystocele repair and midurethral sling surgery at the same time. Thirteen patients (81.3\%) had $\geq$ grade III apical pro-

Table 2. Surgical outcomes of robotic sacrocolpopexy $(n=16)$

\begin{tabular}{lc}
\hline Variable & Value \\
\hline Operation time (min) & $251(236-288)$ \\
Estimated blood loss (mL) & $75(50-150)$ \\
Combined operation & $9(56.3)$ \\
Midurethral sling & $8(50.0)$ \\
Cystocele repair & $1(6.3)$ \\
Conversion to open surgery & $0(0)$ \\
Hospital stay (day) & $4(3-5)$ \\
Uterine/vaginal vault prolapse & \\
Grade 0 & 8 \\
Grade I & 6 \\
Success at last follow-up & $14(100)^{\mathrm{a})}$ \\
Recurrence at last follow-up & $0(0)$
\end{tabular}

Values are presented as median (interquartile range) or number (\%). ${ }^{a}$ Only 14 women were measured by the questionnaire before the operation and at the final follow-up. 
lapse. Stress incontinence was reported in 11 patients (68.8\%; Table 1).

Perioperative outcomes are shown in Table 2. Operation duration was 251 minutes (IQR, 236-288 minutes), and blood loss was estimated to be $75 \mathrm{~mL}$ (IQR, $50-150 \mathrm{~mL}$ ). The median hospital stay was 4 days (IQR, 3-5 days). At the last follow-up, 14 patients underwent POP-Q examination. Treatment success was reported in all patients who presented grade $0(n=8$, $57.1 \%)$ and grade $I(n=6,42.9 \%)$ apical prolapse. As shown in Table 3, improvements in apical prolapse were assessed by POP$\mathrm{Q}$ examination (preoperative $\mathrm{C}$ point, 4 [2-5]; postoperative $\mathrm{C}$ point, $-6[-8$ to -5$] ; \mathrm{P}=0.001$ ). There were statistically significant improvements in anterior and posterior wall prolapse as well as apical prolapse (all P-values $<0.05$ ). No recurrences occurred during follow-up.

Only 14 women completed the PFDI-SF 20 before the operation and at the final follow-up (Fig. 1). Dramatic improvements in all subscales of the PFDI-SF 20, including the total score, POP distress, colorectal-anal distress, and urinary distress inventories, were noted after the operation (all P-values $=0.001$ ). Results of the TSQ showed that 12 of 13 patients (92.3\%) were

Table 3. Measurement of pelvic organ prolapse by using pelvic organ prolapse quantification examination $(n=14)$

\begin{tabular}{lccc}
\hline Point & Preoperative & Postoperative & P-value $^{\mathrm{a})}$ \\
\hline Aa point & $3(1-3)$ & $-2(-2$ to 0$)$ & 0.002 \\
Ba point & $3(3-5)$ & $-2(-2$ to -1$)$ & 0.001 \\
C point & $4(2-5)$ & $-6(-8$ to -5$)$ & 0.001 \\
Ap point & $-2(-2$ to 3$)$ & $-2(-3$ to -2$)$ & 0.016 \\
Bp point & $2(-2$ to 3$)$ & $-2(-3$ to -2$)$ & 0.002 \\
gh & $5(4-6)$ & - & - \\
pb & $3(2-4)$ & - & - \\
tvl & $8(7-9)$ & - & - \\
\hline
\end{tabular}

$\mathrm{Aa}$, point $\mathrm{A}$ anterior; $\mathrm{Ap}$, point $\mathrm{A}$ posterior; $\mathrm{Ba}$, point $\mathrm{B}$ anterior; $\mathrm{Bb}$, point $B$ posterior; $C$, cervix or vaginal cuff; gh, genital hiatus; $\mathrm{pb}$, perineal body; tvl, total vaginal length.

${ }^{a}$ Wilcoxon signed-rank test. "very satisfied" ( $n=6,46.1 \%)$ or "satisfied" ( $n=6,46.1 \%)$ with RSC. However, 1 patient reported that she was "very dissatisfied" with the treatment, although she had grade I apical prolapse at the final follow-up. She had presented with central-type cystocele, which persisted even after the surgery.

An intraoperative complication (i.e., sacral venous plexus injury resulting in massive bleeding and transfusion) was reported in 1 patient who was among the first to undergo RSC. However, there was no case of conversion to open surgery. Postoperative complications were reported in 4 patients but were transient in all patients (Table 4). Bowel injury, ureteral injury, and mesh erosion were not reported in any patients. Of the $5 \mathrm{pa}-$ tients who did not experience stress incontinence before the surgery, 2 reported de novo stress incontinence.

\section{DISCUSSION}

The present study demonstrated that RSC is an effective, reliable, and safe method for apical prolapse repair. At the last fol-

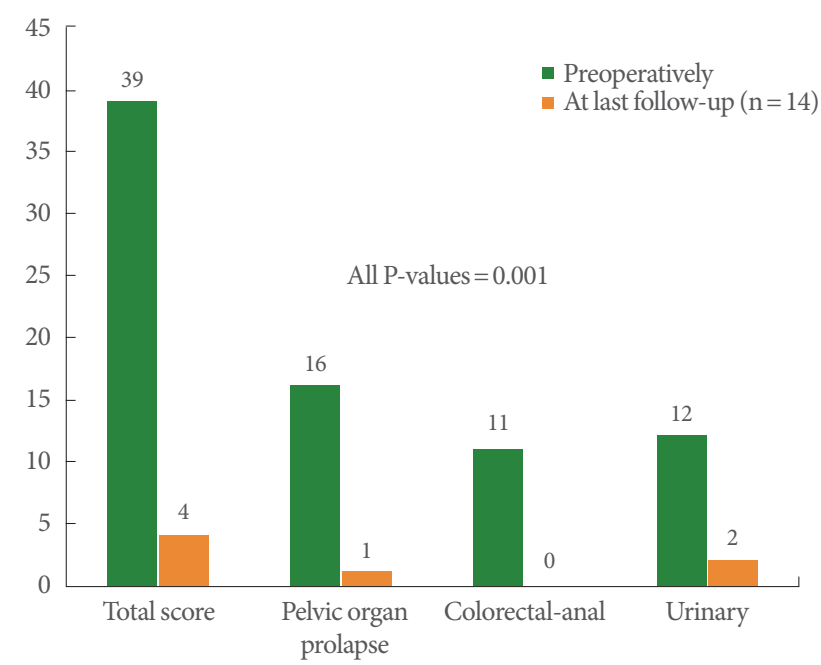

Fig. 1. Measurement of patient-reported outcomes for robotic sacrocolpopexy by using pelvic floor distress inventory-short form 20 .

Table 4. Detailed description of perioperative complications

\begin{tabular}{|c|c|c|}
\hline Complication & No. $(\%)$ & Detail \\
\hline Intraoperative complication & $1(6.3)$ & Sacral venous plexus injury causing massive bleeding \\
\hline \multicolumn{3}{|l|}{ Postoperative complication ${ }^{\mathrm{a})}$} \\
\hline Grade I & $3(18.8)$ & Headache $(\mathrm{n}=1)$, nausea $(\mathrm{n}=1)$, transient nerve palsy $(\mathrm{n}=1)$ \\
\hline Grade II & $1(6.3)$ & Postoperative transfusion $(\mathrm{n}=1)$ \\
\hline
\end{tabular}

${ }^{a)}$ Using Clavien-Dindo complication classification. 
low-up, successful outcome measures based on POP-Q scores were reported in all patients. In addition, most patients (12 of 13) were satisfied with the operation. Only 1 woman who was dissatisfied with the operation presented with persistent centraltype cystocele and underwent cystocele repair approximately 2 years after RSC. Using the validated PFDI-SF 20, the subjective outcomes of RSC were assessed. Improvements in all subscales of the PFDI-SF 20 were confirmed at the last follow-up visit. An intraoperative complication (i.e., sacral venous plexus injury) was reported in only 1 patient who was among the first to undergo RSC. Thereafter, peritoneal dissection to expose the anterior longitudinal ligament on the sacral promontory was performed with the aid of an assistant. By using palpation and devices facilitating tactile sensation, surgeons determined the appropriate sites for dissection and mesh fixation. During the immediate postoperative period of 90 days, no major complications (Clavien-Dindo classification grade III or greater) were reported. To our knowledge, this is the first study to evaluate the surgical outcomes and safety of RSC in Korea.

Since the introduction of RSC, several studies including randomized trials and meta-analyses have reported data on the reliability, efficacy, and safety of this technique over the past decade $[16,17,22]$. The European Association of Urology stated that the surgical outcomes of RSC were favorable, and therefore, this procedure was given a grade A recommendation [23]. Midterm follow-up data in the present study also confirmed the results of previous analyses on the surgical outcomes and safety of this technique. With confirmation of the efficacy and safety of RSC and the rising popularity of robotic surgery, use of this technique for apical prolapse repair might increase in the future. Extrapolating from the experience of robotic surgery in prostate cancer, it is reasonable to assume that RSC may be widely used in the near future.

There has been increasingly widespread use of synthetic mesh in the management of POP, although data on synthetic mesh use are controversial. Recently, the Food and Drug Administration highlighted the complications associated with the transvaginal placement of synthetic mesh for POP treatment [24]. Vaginal shortening, tightening, and pain induced by mesh use and mesh erosion were cited as previously unidentified risks. The use of mesh during transvaginal POP repair may introduce risks that are not present in traditional nonmesh surgery. The prostheses/graft complication classification system proposed by the joint meeting of the International Continence Society/International Urogynecological Association can be used to report and accurately characterize mesh complications [25]. Mesh placed abdominally for POP repair may lead to lower rates of mesh-related complications than transvaginal prolapse repair with mesh [8]. In the present study with a medium duration of follow-up, there were no mesh-related complications such as vaginal pain and contraction and mesh erosion. Abdominal placement of mesh for apical prolapse repair seems to be safe in the current study, although further studies with longer follow-up periods are warranted to confirm this finding. Moreover, before the operation, surgeons should clearly explain the postoperative risks and complications associated with mesh use to the patients.

In the current study, 8 women underwent midurethral sling surgery at the time of RSC, and 2 of 5 patients with negative preoperative prolapse reduction stress testing (PRST) had de novo stress incontinence. De novo stress incontinence after sacrocolpopexy remains controversial, although recent studies have reported an overall de novo stress incontinence rate of about $15 \%-30 \%$ in patients with negative PRST $[26,27]$. As the degree of POP increases, stress incontinence is more likely to be "masked" by kinking of the urethra. Anterior vaginal tensioning intended to reduce bladder prolapse during sacrocolpopexy can result in the flattening of the urethrovesical angle and lead to new-onset stress incontinence [26]. Patients with a high Aa point reduction magnitude were at the highest risk of stress incontinence [26]. On the other hand, the role of the posterior vaginal wall in the development of de novo stress incontinence needs to be clarified. De novo stress incontinence could be a disappointing outcome after RSC in previously continent women. Thus, thorough PRST is needed to identify those patients who may benefit from incontinence surgery at the time of RSC. However, stress incontinence may still occur after RSC even in patients with negative PRST.

Currently, there are 3 methods for the surgical management of the uterus: sacrohysteropexy (uterine preservation), supracervical hysterectomy with sacrocolpopexy, and total hysterectomy with sacrocolpopexy. Of these, concomitant total hysterectomy with sacrocolpopexy should be avoided because it is associated with a relatively high rate of mesh erosion [28]. In our institution, sacrohysteropexy for uterine preservation and sacrocolpopexy are performed in women who had undergone previous hysterectomy. In women considering uterine preservation, careful patient selection is critical. Sufficient counseling with respect to the risks of uterine malignancies or other pathologies associated with uterine sparing should be offered be- 
fore surgery [29,30]. Gutman and Maher [28] presented a list of contraindications to uterine-preserving prolapse surgery. Women who experience specific symptoms and have a history of conditions such as uterine abnormalities, postmenopausal bleeding, and abnormal menstrual bleeding should be referred for gynecologic evaluation and excluded from the uterine-preserving protocol.

Limitations of the current study include its retrospective nature. Some main outcomes including POP-Q measure and PFDI survey may have been missed even in the small series. Moreover, the small sample size limited the statistical power for comparison and outcome evaluation. Furthermore, the relatively short-term follow-up may have been inadequate to evaluate the rates of recurrence and complications such as mesh erosion, which usually occurs after a considerable period of time.

In conclusion, the present study demonstrated that RSC is an efficient and reliable technique for uterine/vaginal vault prolapse repair in women. RSC is associated with few intra- and postoperative complications and may be comparable in surgical safety to ASC or LSC according to midterm follow-up data. In addition, most patients were satisfied with the surgical outcomes of RSC. Thus, RSC might be one of the best surgical options for apical prolapse repair in women.

\section{REFERENCES}

1. Wu JM, Matthews CA, Conover MM, Pate V, Jonsson Funk M. Lifetime risk of stress urinary incontinence or pelvic organ prolapse surgery. Obstet Gynecol 2014;123:1201-6.

2. Samuelsson EC, Victor FT, Tibblin G, Svärdsudd KF. Signs of genital prolapse in a Swedish population of women 20 to 59 years of age and possible related factors. Am J Obstet Gynecol 1999;180(2 Pt 1):299-305.

3. Nygaard I, Barber MD, Burgio KL, Kenton K, Meikle S, Schaffer J, et al. Prevalence of symptomatic pelvic floor disorders in US women. JAMA 2008;300:1311-6.

4. Wu JM, Hundley AF, Fulton RG, Myers ER. Forecasting the prevalence of pelvic floor disorders in U.S. Women: 2010 to 2050. Obstet Gynecol 2009;114:1278-83.

5. Subak LL, Waetjen LE, van den Eeden S, Thom DH, Vittinghoff E, Brown JS. Cost of pelvic organ prolapse surgery in the United States. Obstet Gynecol 2001;98:646-51.

6. Svihrova V, Svihra J, Luptak J, Swift S, Digesu GA. Disability-adjusted life years (DALYs) in general population with pelvic organ prolapse: a study based on the prolapse quality-of-life question- naire (P-QOL). Eur J Obstet Gynecol Reprod Biol 2014;182:22-6.

7. Lane FE. Repair of posthysterectomy vaginal-vault prolapse. Obstet Gynecol 1962;20:72-7.

8. Maher C, Feiner B, Baessler K, Schmid C. Surgical management of pelvic organ prolapse in women. Cochrane Database Syst Rev 2013;(4):CD004014.

9. Choi KH, Hong JY. Management of pelvic organ prolapse. Korean J Urol 2014;55:693-702.

10. Ganatra AM, Rozet F, Sanchez-Salas R, Barret E, Galiano M, Cathelineau X, et al. The current status of laparoscopic sacrocolpopexy: a review. Eur Urol 2009;55:1089-103.

11. Nezhat CH, Nezhat F, Nezhat C. Laparoscopic sacral colpopexy for vaginal vault prolapse. Obstet Gynecol 1994;84:885-8.

12. Paraiso MF, Walters MD, Rackley RR, Melek S, Hugney C. Laparoscopic and abdominal sacral colpopexies: a comparative cohort study. Am J Obstet Gynecol 2005;192:1752-8.

13. Tarr ME, Brancato SJ, Cunkelman JA, Polcari A, Nutter B, Kenton K. Comparison of postural ergonomics between laparoscopic and robotic sacrocolpopexy: a pilot study. J Minim Invasive Gynecol 2015;22:234-8.

14. Di Marco DS, Chow GK, Gettman MT, Elliott DS. Robotic-assisted laparoscopic sacrocolpopexy for treatment of vaginal vault prolapse. Urology 2004;63:373-6.

15. Sajadi KP, Goldman HB. Robotic pelvic organ prolapse surgery. Nat Rev Urol 2015;12:216-24.

16. Serati M, Bogani G, Sorice P, Braga A, Torella M, Salvatore S, et al. Robot-assisted sacrocolpopexy for pelvic organ prolapse: a systematic review and meta-analysis of comparative studies. Eur Urol 2014;66:303-18.

17. Anger JT, Mueller ER, Tarnay C, Smith B, Stroupe K, Rosenman A, et al. Robotic compared with laparoscopic sacrocolpopexy: a randomized controlled trial. Obstet Gynecol 2014;123:5-12.

18. Jeon MJ, Moon YJ, Jung HJ, Lim KJ, Yang HI, Kim SK, et al. A long-term treatment outcome of abdominal sacrocolpopexy. Yonsei Med J 2009;50:807-13.

19. Oh S, Shin SH, Kim JY, Lee M, Jeon MJ. Perioperative and postoperative morbidity after sacrocolpopexy according to age in Korean women. Obstet Gynecol Sci 2015;58:59-64.

20. Sung HH, Park BH, Ryu DS, Lee KS. Recent advances in robotic surgery in female urology. Int J Urol 2013;20:1154-62.

21. Yoo EH, Jeon MJ, Ahn KH, Bai SW. Translation and linguistic validation of Korean version of short form of pelvic floor distress inventory-20, pelvic floor impact questionnaire-7. Obstet Gynecol Sci 2013;56:330-2.

22. Paraiso MF, Jelovsek JE, Frick A, Chen CC, Barber MD. Laparo- 
scopic compared with robotic sacrocolpopexy for vaginal prolapse: a randomized controlled trial. Obstet Gynecol 2011;118:1005-13.

23. Merseburger AS, Herrmann TR, Shariat SF, Kyriazis I, Nagele U, Traxer O, et al. EAU guidelines on robotic and single-site surgery in urology. Eur Urol 2013;64:277-91.

24. Urogynecologic surgical mesh: update on the safety and effectiveness of transvaginal placement for pelvic organ prolapse [Internet]. Silver Spring (MD): U.S. Food and Drug Administration, Center for Devices and Radiological Health; 2011 [cited 2015 Aug 2]. Available from: http://www.fda.gov/downloads/MedicalDevices/ Safety/AlertsandNotices/UCM262760.pdf.

25. Haylen BT, Freeman RM, Swift SE, Cosson M, Davila GW, Deprest J, et al. An International Urogynecological Association (IUGA)/International Continence Society (ICS) joint terminology and classification of the complications related directly to the insertion of prostheses (meshes, implants, tapes) \& grafts in female pelvic floor surgery. Int Urogynecol J 2011;22:3-15.

26. LeClaire EL, Mukati MS, Juarez D, White D, Quiroz LH. Is de novo stress incontinence after sacrocolpopexy related to anatomical changes and surgical approach? Int Urogynecol J 2014;25:1201-6.

27. El Hamamsy D, Fayyad AM. New onset stress urinary incontinence following laparoscopic sacrocolpopexy and its relation to anatomical outcomes. Int Urogynecol J 2015;26:1041-5.

28. Gutman R, Maher C. Uterine-preserving POP surgery. Int Urogynecol J 2013;24:1803-13.

29. Bogani G, Serati M, Cromi A, Ghezzi F. Risk of undiagnosed uterine malignancies at the time of robotic supracervical hysterectomy and sacrocolpopexy. Eur Urol 2015;67:352.

30. Frick AC, Walters MD, Larkin KS, Barber MD. Risk of unanticipated abnormal gynecologic pathology at the time of hysterectomy for uterovaginal prolapse. Am J Obstet Gynecol 2010;202:507.e1-4. 